\title{
Pensar la arqueología desde el sur
}

\author{
Thinking about archaeology from the south
}

\author{
Virginia M. Salerno \\ CONICET - Instituto de Arqueología, Facultad de Filosofía y Letras, UBA \\ Ramon Falcón 2647 Piso 1 Dto. 25 (1406) Capital Federal, Argentina \\ vmasalerno@gmail.com
}

Recibido: 19-06-2012

Aceptado: 13-10-2012

\begin{abstract}
Resumen
Hacia fines del siglo XX, la confluencia de diversos movimientos que incluyeron una amplia gama de agentes, prácticas y usos sociales en relación con la materialidad arqueológica, dio lugar al desarrollo de la Arqueología Pública. En el marco de esta línea de trabajo, se vienen elaborando programas de acción, enfoques analíticos y reflexivos que abordan la relación pasado-presente y el posicionamiento de los arqueólogos en estos procesos. En este trabajo se propone que es necesario abordar la articulación arqueología-sociedad anclándola en las características del universo que le dan sentido. Para discutir este postulado se consideran los procesos históricos que particularizan la Arqueología Pública en América Latina donde el conocimiento arqueológico se construyó como el conocimiento sobre el pasado de "otros", excluidos de "nuestra" historia. Además se propone la existencia de una agenda de problemas comunes que conforman la Arqueología Pública en los países del "Sur geopolítico" (Latinoamérica, Oceanía y África).
\end{abstract}

Palabras Clave: Arqueología Pública. América Latina. Alteridad. Ética Profesional.

\begin{abstract}
By the end of twentieth century, the confluence of various movements which involved a wide range of actors, social customs and practices in relation to the archaeological materiality resulted in the development of Public Archaeology. Within this framework, some programs of action and also reflective analytical approaches have been developed. The last addressed the past-present relationship and the positioning of the archaeologists in these processes. In this paper, it is emphasized the necessity of considering the relation between archaeology and society inside the social and historical features of the universe that give them meaning. With the aim of discussing this assumption, the historical processes that particularize the Public Archaeology in Latin America are examined; in this development, the archaeological knowledge is constructed as knowledge about an 'other' which is not recognized as a part of 'our' history. Finally, a list of problems related to Public Archaeology in the Southern countries (Latin America, Oceania and Africa) is proposed.
\end{abstract}

KE ywords: Public Archaeology. Latin America. Alterity. Professional Ethics.

Sumario: 1. Introducción. 2. Ayer, hoy, era mañana. La Arqueología Pública como línea de investigación. 3. Arqueología y alteridad en América Latina. 4. Pensar la Arqueología Pública en el "Sur". 5. Reflexiones finales. 


\section{Introducción}

La época actual se caracteriza por la intensificación y el aumento en la circulación del conocimiento y la heterogeneidad de los agentes que intervienen en las interpretaciones del pasado (Ruiz Zapatero 2002). Estos movimientos han tenido diferentes causas y duraciones que involucraron una amplia gama de agentes, prácticas y usos sociales en relación con la materialidad arqueológica. Pueden mencionarse tanto a las reivindicaciones de pueblos originarios y afroamericanos para ampliar su participación en la gestión y producción de conocimiento sobre su pasado (Trigger 1992, Johnson 2000), como a los procesos de apropiación y de activación patrimonial con fines comerciales, turísticos, científicos, educativos o empresariales, entre otros (Ballart 1997).

Frente a esta situación, desde el campo arqueológico se han revisado los modos en que conocimientos y referentes materiales adquieren significatividad fuera del campo académico. Estos abordajes se están sistematizando dentro de una línea de investigación que se ha llamado Arqueología Pública (McGimsey 1972, Schadla -Hall 1999, Funari 2004, Merriman 2004, Saucedo-Segami 2006, Segobye 2006, Almansa Sanchez 2011, Dansac 2012). Esta línea de trabajo no sólo trata sobre productos arqueológicos (programas educacionales, materiales de transferencia, actividades museísticas, etc.) sino también sobre procesos de negociación y creación de sentidos en torno a la materialidad arqueológica (Funari 2004, Merriman 2004, Segobye 2006). Se incluye una diversa gama de temáticas entre las que pueden mencionarse la consideración de estrategias comunicativas por parte de los investigadores; la investigación de representaciones sociales de la arqueología en el presente (Hall 2004, Hernández 1997, Lemaitre y Schall 2009, Salerno y Pupio 2009, entre otros); y el estudio de los procesos de transformación de los referentes del pasado en mercancías o en lugares emblemáticos que, como parte del patrimonio cultural, legitiman ciertas narrativas sobre otras (Ballart 1997, Querol 2001, Ruiz Zapatero 2002, Gnecco 2004, entre otros). En todos los casos, la reflexión sobre las acciones destinadas a generar formas de comunicación, diálogo e interconsulta con distintos grupos sociales, se relaciona con imperativos éticos y responsabilidades derivadas de la arqueología entendida como una profesión social (Merriman 2004).

Desde este enfoque la ciencia es entendida como una actividad que se lleva a cabo en múltiples contextos sociales (Echeverría 1998). De este modo se diluye el ambiguo límite entre teoría y práctica, dualismo que remite a la dicotomía entre ciencia pura y ciencia aplicada del pensamiento moderno. Partien- do de esta concepción, en este trabajo se discute que la Arqueología Pública tiene características distintivas dependiendo de los procesos históricos a partir de los cuáles el estudio de la materialidad arqueológica se organizó como actividad social. Con este fin, primeramente se presentan algunas consideraciones teóricas sobre el desarrollo de esta línea de investigación, para luego abordar las particularidades de la Arqueología Pública en las sociedades latinoamericanas. Finalmente se propone una serie de aspectos comunes a los países del "Sur geopolítico" (Latinoamérica, Oceanía y África) donde el legado de las diversas trayectorias coloniales conllevaron la construcción de relatos históricos duales que rechazan la diferencia e invisibilizan la desigualdad (Lander 2000, Lahiri et al. 2007).

\section{Ayer, hoy, era mañana. La Arqueología Públi- ca como línea de investigación.}

Hacia fines del siglo XX la confluencia de una serie de procesos sociales, económicos y políticos generaron modificaciones sustanciales en el modo en que la arqueología y los materiales arqueológicos adquieren significatividad en el presente. Para la arqueología fueron significativos los movimientos de las ciencias sociales que discutieron los sentidos del pasado en el presente (Berger y Luckman 1968, Hobsbawn y Ranger 1988, Lowenthal 1998), las luchas de comunidades indígenas y otras minorías sociales por participar en la interpretación de su propio pasado (Hirst 1997, Johnson 2000, Ayala et al. 2003, Fernández Murillo 2003, Byrne 2004, Curtoni 2004, Fernández 2005, McNiven y Russell 2005, Segobye 2006) y los movimientos de capital que llevaron a la definición de los materiales arqueológicos como bienes culturales, con nuevas modalidades de exposición y un creciente proceso de mercantilización de los mismos (Merriman 2002, Ruiz Zapatero 2002, Almansa Sánchez 2011). En conjunto, estos procesos dieron lugar a nuevas formas de entender los vínculos entre pasado y presente (Ballart 1997) y entre ciencia y sociedad (Salomón 2008). En este contexto los arqueólogos formularon interrogantes y expectativas en torno a su práctica, dando lugar a los desarrollos de la Arqueología Pública.

Entre los nombres que se han ensayado para designar esta nueva línea de indagación, la expresión Arqueología Pública se impuso a pesar de las ambigüedades que se derivan en torno al adjetivo. Este es el nombre de las primeras revistas especializadas totalmente dedicadas al tema: Public Archaeology desde el año 2000, revista internacional producida desde la Universidad de Londres (University Co- 
llege London), y desde el año 2006 Arqueología Pública, revista de la Universidade Estadual de Campinas (Brasil). Además de estas ediciones, las discusiones sobre este tema tienen amplia visibilidad en los espacios virtuales de intercambio académico $^{1}$. En estos se presentan recursos y actividades para la comunicación entre los arqueólogos y el público, se discuten los resultados de diversas experiencias comunicativas y con menor frecuencia, se incluyen reflexiones teóricas sobre la relación entre arqueología y sociedad. A diferencia de los ámbitos de discusión tradicionales de comunicación científica, los espacios virtuales tienen costos menores, mayor flexibilidad y rapidez para dar visibilidad a las nuevas ideas (Romanos de Tiratel 2008). Por estos motivos, la información que circula en ellos resulta un indicador interesante para pensar en la emergencia de nuevos problemas de investigación. En congruencia, las narrativas sobre la arqueología y los textos de difusión incluidos en estas páginas sintetizan, reorganizan y presentan conocimientos a una variedad de audiencias que incluyen la comunidad disciplinar de los propios autores (Hochadel 2009), contribuyendo de esta manera a la formación de consenso sobre las teorías en discusión dentro del campo disciplinar (Echeverría 1998, Hochadel 2009).

En los variados abordajes de la Arqueología Pública pueden advertirse dos ejes a partir de los cuales se construyen sentidos en torno a lo "público": como espacio de interacción de la sociedad civil y/o como parte de la esfera del Estado (Merriman 2004, Almansa Sánchez 2011). Ambos remiten a dos matrices conceptuales que formaron parte de los debates analíticos sobre esta categoría dentro de las ciencias sociales (Minteguiaga 2008). En el primer caso, se trata de un enfoque centrado en la historia de los espacios y esferas de debate e intercambio comunicacional. En el segundo caso, la relación entre lo público y lo estatal se organizó en vínculo con el estudio de las políticas públicas (Minteguiaga 2008). Ambas formas de entender lo público no deberían considerarse excluyentes entre sí, en tanto se trata de un espacio complejo que se sustenta a la vez en:

"la capacidad asociativa, participación, deliberación y auto-organización de la sociedad civil, como en la universalidad que el Estado debe asegurar para tornar efectiva la promesa democrática" (Iazzeta 2008:50).

Aplicadas a la arqueología, las distintas aproximaciones a lo público conllevan diversos posicionamientos sobre el lugar de las organizaciones privadas y del Estado en la investigación, protección y comunicación del conocimiento arqueológico.
En este sentido cabe preguntarse a quiénes representan los materiales arqueológicos (Johnson 2000, Funari 2004, Mapunda y Lane 2004, entre otros). Por su parte, los estudios sobre los procesos de objetivación de la materialidad arqueológica en términos patrimoniales, nos recuerdan las relaciones de poder que median la definición de límites entre especialistas y no especialistas, entre arqueólogos y público (Ballart 1997, Londoño 2003, entre otros).

En consonancia con estas ideas, Dansac (2012) menciona tres ejes de discusión que fundamentan el desenvolvimiento de la Arqueología Pública:

“a) la exclusión histórica que ha tenido la gente en la construcción y presentación del patrimonio cultural, b) a la falta de reflexión en las implicaciones socio-económicas y políticas que conlleva el estudio o conservación de un sitio arqueológico para las comunidades próximas, c) y a la nula o escasa comunicación con la gente para la cual se desentierra y explora diariamente el pasado".

Estos ejes remiten a recorridos históricos e institucionales que hicieron posible la investigación arqueológica en un determinado lugar. Se incluyen aquí relaciones humanas que se entretejieron articulando ideas, objetos, presente y pasado. En este sentido, lo interesante de esta propuesta es que pone en primer plano los procesos históricos a partir de los cuáles el estudio de la materialidad arqueológica se organizó como actividad social y nos recuerda que no es posible pensar la articulación arqueologíasociedad sin anclar estas indagaciones en las características del universo que le da sentido.

Desde esta perspectiva se pueden analizar las tempranas reflexiones sobre las incumbencias de la Arqueología Pública en las dos primeras revistas especializadas sobre el tema mencionadas anteriormente. En el editorial del primer número de la revista inglesa Public Archaeology, Neal Ascherson (2000) expresa que la Arqueología Pública surge en Gran Bretaña asociada a los movimientos postsesenta cuando esta disciplina "abre" su campo. Asimismo, el autor relaciona dicha apertura con la ética profesional; con una serie de actividades relacionadas con la interpretación y manejo del pasado en el presente y con el reconocimiento de responsabilidades sociales y políticas derivadas del estatus profesional de la arqueología. Seis años después, en el editorial del primer número de Arqueología Pública, editado en el Núcleo de Estudios Estratégicos de la Universidad Estadual de Campinas de San Pablo, Pedro P. Funari y Erika M. Robrahn-González (2006) proponen que la expresión Arqueología Pública puede entenderse como una acción con el pueblo, una ciencia aplicada en beneficio de las 
comunidades y de los diferentes grupos sociales, aludiendo con esto a los procesos de negociación y a los conflictos derivados de los procesos de interpretación del pasado.

Se trata de dos definiciones cuyas diferencias se derivan de dos geopolíticas: un país colonizador, un continente colonizado (Escobar 1998, Lander 2003). En ambas propuestas el reconocimiento del proceso de profesionalización de la disciplina y las regulaciones éticas de la acción son dos dimensiones que confluyen en la delimitación del especialista y sus funciones frente a otros no especialistas. Sin embargo hay diferencias en la manera en que se proponen estas delimitaciones y funciones. En el primer caso se pone el acento en el proceso de reconocimiento del arqueólogo como una actividad más en el presente y de los objetos arqueológicos como su producto. En el segundo, la discusión de la articulación arqueología-sociedad incluye el controvertido rol de la arqueología en América Latina y se construye en torno a una toma de posición con respecto al rol del profesional. Este posicionamiento busca comprometerse con la diversidad de trayectorias históricamente excluidas e invisibilizadas mediante las narrativas construidas desde la arqueología en este continente (Díaz-Andreu 1999; Dussel 1994).

De estas diferencias se desprenden no sólo distintas formas de abordajes entre distintos centros de estudio, también se incluyen diferentes criterios para la definición de problemas y modos de resolverlos. Por ello consideramos que los problemas susceptibles de ser investigados en el marco de la Arqueología Pública son inseparables de las trayectorias históricas a través de las cuales en un determinado lugar la arqueología se legitimó como actividad social, ya que abordar la arqueología y sus objetos en términos de productos y no en términos de procesos de construcción social y simbólica, implicaría desconocer las relaciones de desigualdad a través de las cuales esta disciplina se constituyó (Galison 1991, Bourdieu 2003). Siguiendo esta línea de pensamiento, en el próximo apartado se postulan una serie de consideraciones que nos permitirán contextualizar los estudios de la Arqueología Pública en América Latina.

\section{Arqueología y alteridad en América Latina}

Para pensar las múltiples formas de articulación de la arqueología en las sociedades latinoamericanas, dos dimensiones ineludibles vinculadas a su trayectoria histórica son la situación colonial y los movimientos nacionalistas independentistas de principios del siglo XIX (Lumbreras 1974, Díaz-Andreu
1999). Existe acuerdo en que las nacientes ciencias del siglo XIX (filología, arqueología, historia, etnología, antropología, paleontología) produjeron un imaginario sobre el mundo social de los dominados. Este se vincula con el pensamiento de la Ilustración y fue explicado por Dussel (1994) como el mito eurocéntrico de la modernidad que afirma la existencia de un proceso histórico lineal en el cual Europa, y con ella la ciencia moderna y el capitalismo naciente, se proponen como lugar privilegiado de enunciación y producción de conocimientos. A partir de este imaginario no sólo se legitimó el poder imperial en el nivel económico y político sino que se construyeron los presupuestos epistemológicos de las nacientes ciencias sociales, entre ellas la arqueología (Dussel 1994, Mignolo 1995, Lander 2003).

En América Latina las estructuras científicas que promovieron el estudio del pasado se fundaron en asociación con los ideales modernizadores de las elites gobernantes de la segunda mitad del siglo XIX (Saldaña 1993). Las investigaciones arqueológicas contribuyeron a la elaboración de un relato histórico que legitimó los proyectos nacionalistas de aquéllas (ver Fernández Murillo 2003 para Bolivia, Gnecco 2004 en Colombia, Verdesio 2004 en Uruguay, Piñon 2008 en Brasil, Podgorny 2009 en Argentina). En ese momento los museos y "Sociedades Amigas", instituciones que avalaron la actividad científica en general y la arqueología en particular (Arenas 1989/90), se caracterizaron por las relaciones de dependencia con los centros hegemónicos y el objetivo imperioso de inserción y visibilidad internacional que perseguían las dirigencias locales (Mantegari 2002). Es relevante mencionar que en el impulso de estas instituciones, tuvieron una participación determinante diversos estudiosos y especialistas extranjeros. Estos agentes delinearon la selección de estrategias y criterios académicos y organizativos de estas nuevas instituciones para la actividad científica, principalmente asociadas al desarrollo del "saber natural" (Mantegari 2002).

En este contexto, el marco epistemológico para los estudios arqueológicos se organizó con base en los estudios de la arqueología prehistórica desarrollada a fines del siglo XIX (Gnecco 2004, Haber 2004, Nastri 2005, Londoño 2007, Piñon 2008, Pegoraro 2009, Meneses Pacheco 2010, Carrizo 2010). La novedad de los estudios prehistóricos residió en la aceptación de que la antigüedad del ser humano no podía limitarse a las propuestas bíblicas y su posible convivencia con animales extintos, tesis que se sostuvo gracias a la confluencia de los conocimientos geológicos, los hallazgos arqueológicos y las ideas plasmadas por Darwin sobre el origen de las especies (Daniel 1973). Este enfoque promovió 
el estudio del pasado humano remoto utilizando como marco interpretativo el sistema cronológico de las tres edades (Edad de Piedra, Edad de Bronce y Edad de Hierro), definidas principalmente en base a referentes europeos, para explicar y estudiar los hallazgos arqueológicos de todo el mundo (Daniel 1973, Podgorny 2009).

Es sabido que en este esquema universal la definición de las sociedades del pasado se realizó en contraposición con las del presente, construyendo discursos de alteridad donde el "nosotros" estaba representado por la sociedad europea occidental. Los "otros" representaron lo incompleto, el camino por el cual la humanidad toda llegaría a las características del "nosotros" (Fabian 1983, Hernando 2006). A la vez, para explicar la diferencia entre culturas se utilizaron las ideas racistas del evolucionismo lineal, conformando un alineamiento teórico entre arqueología y etnología europea que Trigger (1992) caracterizó como "síntesis imperial". Con fines comparativos se promovió el intercambio de novedades y datos a través de redes privadas y públicas que contribuyeron a la formación de un sentido de pertenencia entre estudiosos del tema dentro de un esquema de producción de conocimientos definido como universal (Trigger 1992, Piñon 2008, Podgorny 2009).

Los discursos forjados en estas relaciones de dependencia (teórica y política) confluyeron en la forma en que los estudiosos del pasado americano fueron organizando sus interpretaciones y prácticas arqueológicas, a la vez que se distinguieron de otros sujetos sociales y otras formas de interpretar el pasado. En un ámbito de disputas sobre las interpretaciones del pasado, los arqueólogos latinoamericanos construyeron su legitimidad en contraposición con dos frentes. Por un lado, los no-especialistas locales, que fueron desplazados de su derecho de enunciación con respecto a su propia historia, pero que eran portadores de saberes con relación a los lugares donde se encuentran los materiales arqueológicos. Por el otro, los especialistas de los centros hegemónicos, con quienes se mantuvo una relación de dependencia teórica en función de las estructuras de saber colonial (Nastri y Ferreira 2010). A su vez el conocimiento que produjeron los arqueólogos en América Latina tendió a disminuir la importancia de los logros tecnológicos, culturales y políticos de las culturas americanas, atribuyéndolos a la influencia de otras civilizaciones o negándoles profundidad histórica. De modo tal que las interpretaciones sobre los materiales arqueológicos se realizaron en pos de negar la historia a los indígenas americanos, a la vez que se los insertaba en un esquema temporal europeo, concebido como natural y universal (Haber 2004, Londoño 2007). En este esquema los datos etnológicos y documentales prevalecieron como fuente para interpretar y explicar los materiales arqueológicos (Fagan 1972, Politis 1995, Nastri 2005, Nastri y Ferreira 2010).

Como resultado de estos procesos, el pasado americano como objeto de estudio de la arqueología fue discursivamente naturalizado mediante la construcción de las nociones de tiempo y alteridad (Haber 2004). Al respecto resulta esclarecedora la relación que propone Augé (1994) entre antropología e historia en torno a la naturaleza de su objeto. Mientras la historia puede pensarse como una disciplina que se desplaza en el tiempo para conocer el pasado de sociedades próximas, la antropología estudia el presente de sociedades diferentes, teniendo como eje el desplazamiento espacial (Fabian 1983). Por ello, en gran parte de la arqueología americana puede considerarse un doble desplazamiento: en el espacio y en el tiempo. Este sería el caso de las investigaciones que abordan el estudio de procesos poblacionales que forman parte de la historia de "otros", excluidos de las identidades nacionales (Wolf 1987, Londoño 2007).

Esta dualidad de origen está presente en la manera en que los agentes sociales dan sentido a la información sobre el pasado, generando representaciones diversas sobre el conocimiento arqueológico y la narrativa histórica. A su vez es parte del modo en que los arqueólogos se posicionan como productores de conocimiento en relación con otros agentes sociales (Haber 1993). Por estos motivos estamos de acuerdo con Joan Gero (2000) en que el desarrollo de la arqueología en una trayectoria de profesionalización exclusiva, organizada de forma internacional, redujo la diversidad de agentes autorizados para estudiar el pasado e impuso las interpretaciones producidas en los centros dominantes. Para dar perspectiva a esta discusión, en el próximo apartado retomamos cómo estos aspectos impactaron en las formas en que se comenzó a desarrollar la Arqueología Pública en América Latina, incorporando la comparación con otros países del "Sur geopolítico" (Latinoamérica, Oceanía y África) donde las trayectorias históricas coloniales a pesar de sus diferencias, permiten discutir problemas comunes (Lander 2000, Lahiri et al. 2007).

\section{Pensar la Arqueología Pública en el "Sur"}

En los países de África y Oceanía se ha señalado la importancia de los movimientos de descolonización -que dieron lugar a la emergencia de formaciones estatales autónomas- para el desarrollo de una práctica arqueológica profesional que respondiera a intereses locales (Mapunda y Lane 2004, Parker 
Pearson y Ramilisonina 2004, McNiven y Russell 2005, Segobye 2006). En América Latina fue primordial la estabilidad derivada de la instauración de regímenes democráticos durante el último cuarto del siglo pasado (Politis 1995). En esos contextos se puso en primer plano la necesidad de una reorganización política, social, económica y educativa. En lo que refiere a la arqueología, estos cambios llevaron a la discusión sobre su proyección social en distintos niveles (normativo, educativo, trabajo participativo).

En todos los casos, en los planos normativos e institucionales se hizo patente la necesidad de reformas para actualizar los marcos de protección, gestión e investigación de los materiales arqueológicos naturalizados en términos de patrimonio cultural con dispares niveles de efectividad (Durán Serrano 2001, Piazzini 2001, Richardson III 2001, Endere y Rolandi 2007, Guraieb y Frère 2008). Es destacable que en algunos casos, como el de Bolivia y Argentina, estos movimientos desembocaron en el establecimiento de un modelo de nación pluricultural (Podgorny 1999, Lima Tórrez 2003). En otros lugares, como Uruguay y Colombia, se buscó generar las condiciones institucionales para facilitar la existencia de proyectos globales que incluyeran la comunicación de resultados (López y Reyes 1994, Curbelo y Cabrera Pérez 1992, López Mazz 2004).

En cuanto a los espacios educativos, se establecieron críticas a los sesgos de las narrativas de las historias nacionales oficiales reproducidas mediante la enseñanza formal (Podgorny 1999, Funari 1994, Mapunda y Lane 2004, Parker Pearson y Ramilisonina 2004, Kojan y Angelo 2005, Segobye 2006, Tantaleán 2010). De la misma manera, los museos fueron objeto de diversos análisis relacionados con la conservación de las colecciones, las representaciones de las narrativas museográficas y los modos de participación que éstos posibilitaban (Odak 1985, Téllez 2002, Fernández Murillo 2003, Godoy Gallardo et al. 2003, Parker Pearson y Ramilisonina 2004, Angelo 2005). Como correlato de estas revisiones, aumentó la creciente preocupación y participación de equipos de arqueología en la organización de actividades educativas de perfeccionamiento docente y en la reformulación de guiones museográficos en los ámbitos locales (Odak 1985, Quintana 1999, Pupio y Visotsky 2001, Londoño y Guatavita 2003). Estos trabajos pusieron en primer plano el lugar de los museos como mediadores entre comunidad e investigación y como espacio público donde el conocimiento puede ser aprehendido. La relación entre los museos y la comunidad comenzó a ser replanteada mediante la formulación de programas que promovieron diversas formas de participación (Delgado Cerón y Mz-Recaman 1994,
Moreno de Giraldo 1994, Gándara Vázquez 1999, Conforti et al. 2007, Piñon 2008).

La relación museos-arqueología también ha sido estudiada desde una perspectiva histórica para considerar la articulación de estos espacios con la investigación y formación disciplinar (Mazel y Ritchie 1994, Podgorny 2004, MacDonald y Schaw 2004, Roca 2008). Además, esta perspectiva histórica permitió revisar el lugar de estas instituciones en los procesos de profesionalización y de distinción entre arqueólogos y aficionados (Ucko 1994, Pupio 2005, Piñon 2008). En ambos casos los estudios evidenciaron la compleja trama en la que se entrelazan los vínculos existentes entre la producción de conocimiento arqueológico y diversos agentes sociales.

En conjunto, puede decirse que estos estudios y propuestas más que acciones continuas, homogéneas y lineales, son tácticas fragmentarias en geopolíticas signadas por la organización burocrática y la escasez de recursos humanos y financieros para la protección y gestión de la información y de las colecciones arqueológicas. Entendiendo "protección" en un sentido amplio que incluye desde aspectos relacionados con las condiciones de conservación de las colecciones en los museos públicos y espacios de investigación, hasta los mecanismos institucionales para evitar el tráfico ilegal de materiales arqueológicos. Así por ejemplo, en Costa Rica la normativa adoptada en el año 1999 tendió a disminuir las protecciones estatales de los materiales arqueológicos a favor de la incorporación de la práctica privada sin controles éticos del quehacer arqueológico (Calvo Mora et al. 2001). La falta de códigos de ética y marcos de regulación de la práctica profesional de los arqueólogos también se ha destacado como un inconveniente en Argentina (Endere 2001), en el este y sur de África (Mapunda y Lane 2004, Segobye 2006) y en Chile (Durán Serrano 2001), entre otros.

Para finalizar, proponemos una reflexión en relación con los problemas derivados de la "participación". En el contexto argentino, el creciente interés de distintos agentes en las comunidades locales por la materialidad arqueológica anunciado por Endere (2001) se relacionaba con dos tendencias. Por un lado, las firmes demandas de participación de grupos indígenas en la gestión de sus referentes del pasado y por el otro, la proliferación de emprendimientos privados destinados a explotar sitios arqueológicos como recursos turísticos. Hoy puede decirse que ambos fenómenos se han profundizado y se encuentran lejos de ser un caso particular de Argentina. Por una parte, y como ya se mencionó previamente, desde el último cuarto del siglo pasado, los movimientos indígenas en un particular contexto histórico promovieron importantes revisiones 
epistemológicas y ontológicas dentro del campo arqueológico. En los países del "Sur geopolítico" la visibilidad de estos grupos como sujetos de derecho dio lugar a movimientos políticos particularmente complejos, puesto que se enlazan con las trayectorias históricas de exclusión y marginación coloniales. Estos movimientos también conllevaron reformulaciones de los marcos legales, políticos y económicos, principalmente relacionados con la restitución de recursos económicos y simbólicos (Molinari 2000, Durán Serrano 2001, Ayala et al. 2003, Fernández Murillo 2003, Parker Pearson y Ramilisonina 2004, Endere 2005, Fernández 2005, Massa 2008, Ramos 2009).

Por otra parte, en el aprovechamiento de la materialidad arqueológica con fines turísticos, un lugar común en los países del "Sur geopolítico" se refiere a los limitados beneficios para las comunidades locales y un uso no sustentable de estos recursos (Vargas Arenas 1990, Endere 2002, Lima Tórrez 2003, Nielsen et al. 2003, Crespo 2006, Segobye 2006, Endere y Rolandi 2007, Onetto et al. 2008). A esto se suma que en las últimas décadas el potencial valor turístico de la materialidad arqueológica fue un eje de las políticas de desarrollo regional bajo el lema que sustenta el binomio turismo=desarrollo, haciendo de su puesta en acción un desafío para los arqueólogos comprometidos con las realidades donde trabajan (Gil García 2005, Nuñez 2006, Segobye 2006, Guraieb y Frère 2008). Además, puede agregarse que el turismo es sólo una de las formas de actividad económica legitimadas bajo la fórmula del desarrollo que afectan a la materialidad arqueológica. Desde la década de 1990, en el continente americano se ha dado cuenta de los problemas derivados por la implementación de megaproyectos privados dirigidos a la explotación de recursos naturales (Ratto 2002, Guraieb y Frère 2008, Jofré 2010) y el desarrollo de grandes obras de infraestructura (oleoductos y acueductos, represas, entre otros).

Estos problemas nos recuerdan que la arqueología es ante todo una actividad cuyo sentido es parte del presente y nos advierte sobre la importancia de tomar una posición frente a determinados procesos sociales. En ese camino consideramos prioritario incluir en la investigación arqueológica la revisión de prácticas y categorías utilizadas para la interpretación, producción y gestión del conocimiento sobre el pasado en pos de generar nuevos marcos que posibiliten el reconocimiento de distintos agentes e intereses comunales. Es por ello que entendemos el trabajo de investigación y acción incluido dentro de la Arqueología Pública como una vía para comprender cómo y en qué universos de sentido la información arqueológica se integra en el presente, con vistas a lograr información de base para proyectar líneas de acción que promuevan el diálogo y uso local del conocimiento arqueológico (Vara y Mendoza 2004).

\section{Reflexiones finales}

En este trabajo nos propusimos llamar la atención sobre la necesidad de revisar el quehacer de la Arqueología Pública, en el marco de los contextos sociales e históricos particulares que le dieron lugar. Tal como se observó, los diferentes contextos históricos no sólo conllevan diversos sentidos de lo público, sino también disímiles criterios para la definición de problemas y modos de resolverlos. Estos se vinculan con los recorridos históricos e institucionales que hicieron posible la investigación arqueológica en un lugar; y la presencia de instituciones y agentes sociales que en determinados momentos disputaron/disputarán el valor simbólico del precedente.

En el caso de los países del "Sur geopolítico" puede decirse que como parte de los legados de la experiencia colonial, los relatos históricos se erigieron a partir del rechazo de la diferencia, invisibilizando la desigualdad. Diferencias que en el estudio del pasado se concretan en la dualidad señalada entre arqueología e historia. Con el transcurso del tiempo, los procesos de exclusión física y/o simbólica de grupos nativos ocurrieron junto con movimientos migratorios y la elaboración de nuevas subjetividades en el marco de nuevos contextos sociales. Por estos motivos, en la actualidad coexisten y se desarrollan subjetividades a partir del entrelazamiento de prácticas fragmentadas y contradictorias que combinan estas diversas trayectorias históricas, conformando lo que Mignolo (2003) denominó la "doble conciencia criolla", o lo que Mia Couto (2003) observó en Mozambique como "ciudadanías diferentes".

Dentro de este esquema, la organización de la materialidad arqueológica en el presente se inscribe en el marco de distintos procesos de identificación y categorización de sujetos, tradicionalmente homogeneizados en la categoría "identidad" (Handler 1996, Brubaker y Cooper 2001). Se trata de procesos de apropiación del conocimiento sobre el pasado arqueológico ligados a situaciones concretas y que incluyen relaciones identitarias, económicas y políticas, entre otras (Salerno 2011-2012). Aquí nos interesa destacar que el modo en que la relación arqueología-sociedad se construye puede variar entre un abanico de posibilidades que van desde relatos fetichistas, ahistóricos, que legitiman situaciones de dominación, a la producción de relatos críticos, historizantes y por tanto no naturalizados. 
Estos últimos son los relatos que permitirían fundar una práctica de la "liberación" (Dussel 1983). Ésta, en tanto que transformación de la realidad, es una posibilidad que descansa en la concepción de la historia como un proceso dinámico, continuo y dependiente de las acciones humanas (Lander 2000).

Estas particularidades que afectan a las formas de construir sentidos en torno a la arqueología en el presente, son parte de las contradicciones a partir de las cuales se erige la efectiva puesta en práctica de la arqueología en los países del "Sur geopolítico". Las mismas se relacionan tanto con el lugar de los profesionales y el discurso que se sustenta, como con el contexto social en que se producen y distribuyen los conocimientos (McGuire y Navarrete 1999). Por estos motivos, la reflexión sobre los aspectos éticos debe ser un elemento necesario para el desenvolvimiento de la Arqueología Pública. Entendiendo la ética como una faceta dentro del proceso de investigación que aspira a la igualdad dentro de las relaciones de saber y poder asimétricas que se establecen entre los agentes involucrados (Rance y Salinas 2001). Así, la autocrítica con respecto al rol histórico de la arqueología y la búsqueda de caminos para ampliar la participación y contribuir a visibilizar a poblaciones históricamente no visibles, en principio pueden considerarse móviles teóricos que se enmarcan con esos ideales.

\section{Notas}

1. Para un análisis crítico sobre las formas de comunicación entre arqueólogos y públicos a través de estos espacios puede consultarse McDavid (2004). Algunas de las páginas dedicadas específicamente al tema son:

http://www.flpublicarchaeology.org/; http://www.publicarchaeology.eu/; http://publicarchaeology.blogspot.com/; http://www.arqueologiapublica.es/; http://www.saa.org/publicftp/PUBLIC/home/home.html; http://www.arqueologiapublica.com.br.

https://arkeopatias.wordpress.com

\section{Agradecimientos}

Este trabajo fue realizado como parte de una beca de postgrado financiada por CONICET y en el marco de dos proyectos mayores: UBACyT EXP-UBA No 18829/2010 (2011-2014) y PICT 2010-1517 (2011 - 2013). Al Dr. Mariano Ramos, la Dra. María Isabel González, la Dra. Alejandra Pupio y la Lic. María Magdalena Frère por sus comentarios y sugerencias que nos permitieron enriquecer este manuscrito. A la Dra. Paula Escosteguy por su colaboración en la traducción del resumen.

\section{REFERENCIAS BibLIOGRÁficas}

AlmANSA SÁncheZ, J. (2011): Arqueología para todos los públicos. Hacia una definición de la arqueología pública "a la española". ArqueoWeb - Revista sobre Arqueología en Internet 13:87-107 [URL: http://www.ucm.es/info/ arqueoweb/pdf/13/almansa.pdf] Acceso el 19/04/2012.

AngELo, D. (2005). La arqueología en Bolivia. Reflexiones sobre la disciplina a inicios del siglo XXI Arqueología Suramericana - Arqueología sul-americana, Vol 1(2):185-212.

ArenAs, P. (1989/90): La antropología n la Argentina de fines del siglo XIX y principios del XX. Runa 19: 147160.

Ascherson N. (2000): Editorial. Public Archaeolgy 1:1-4.

Augé, M. (1994): Hacia una antropología de los mundos contemporáneos. Gedisa, Barcelona.

Ayala P.; Avendaño, S.; CÁrdenAs, U. (2003): Vinculaciones entre una arqueología social y la comunidad indígena de Ollagüe (Región de Antofagasta, Chile). Chungara, Revista de Antropología Chilena, 35(2): 275-285

BALLART, J. (1997): El patrimonio histórico y arqueológico: valor y uso. Ariel, Barcelona.

Berger P.; LuKMAn, T. (1968 [1983]): La construcción social de la realidad. Amorrutu, Madrid.

Bourdieu, P. (2003): El oficio del científico. Ciencia de la ciencia y reflexividad. Curso del College de France 2000 - 2001. Anagrama, Barcelona.

Brubaker, R.; CoOper, F. (2001). Más allá de la "identidad”. Apuntes de investigación del CECYP, 7:30-66. 
Byrne, D. (2004): Archaeology in reverse, The flow of Aboriginal people and their remains through the space of New South Wales. En Merriman 2004: 240 -254.

Calvo Mora, M., Arias Quirós, A.C.; Troyo Vargas, E. (2001): La protección, conservación y divulgación del patrimonio arqueológico: el caso de Costa Rica. Revista de Arqueología Americana, 20: 9-30.

CArrizo, S. R. (2010): Exploraciones arqueológicas en la construcción del territorio tucumano de fines del siglo XIX y principios del XX. En Nastri y Ferreira 2010: 55-76.

Conforti, E.; Endere, M.L.; Errobidart, A. (2007): El patrimonio arqueológico en relación a la educación no formal, la comunicación y la exhibición. El caso del Museo Municipal “José Mulazzi” de la ciudad de Tres Arroyos, Provincia de Buenos Aires. V Jornadas Técnicas sobre Conservación, Exhibición y Extensión Educativa en Museos, Río Cuarto, Córdoba.

Couto, M. (2003): Economia: A Fronteira da Cultura [URL: http://bazongadakilumba.blog-city.com/read/169355. htm].

CRESPo, C. (2006): Entre el 'deber'y el 'derecho': patrimonio arqueológico y obligaciones sociales en Patagonia Argentina. Intersecciones en Antropología, 7: 62-75.

Curbelo C.; Cabrera Pérez, L. (1992): Patrimonio y arqueología en Uruguay: hacia el reconocimiento de un pasado olvidado. Arqueología en América latina hoy (G. Politis ed.), Presencia, Santa Fe de Bogota: 45-56.

Curtoni R. P. (2004). La dimensión política de la Arqueología: el patrimonio indígena y la construcción del pasado. Aproximaciones contemporáneas a la arqueología pampeana: perspectivas teóricas, metodologías analíticas y casos de estudio (G. Martínez, M. A. Gutiérrez, R. Curtoni, M. Berón y P. Madrid, eds.). UNCPBA, Facultad de Ciencias sociales, Olavaria: 437- 450.

Daniel, G. (1973): El concepto de prehistoria. Editorial Labor, Buenos Aires.

DANSAC, Y. (2012): Diálogos y vinculaciones entre el arqueólogo y la comunidad en cinco continentes: una invitación a la arqueología pública. Blog. [URL: https://arkeopatias.wordpress.com/2012/02/04/dialogos-y-vinculaciones-entre-el-arqueologo-y-la-comunidad-en-cinco-continentes-una-invitacion-a-la-arqueologia-publica/] Acceso el 5 de mayo 2012.

Delgado Cerón, I.; Mz-Recaman, C.I. (1994): The Museum comes to School in Colombia: Teaching Packages as a Method of Learning. The presented past. Heritage, Museums and Education, (P. Stone, P. y B. Molyneaux, eds.), Routledge, Londres: 148-158.

DíAz-Andreu, M. (1999): Nacionalismo y Arqueología: del Viejo al Nuevo Mundo. Revista do Museu de Arqueologia e Etnologia, Anais da I reuniao Internacionacional de Teoria Arqueologica na America do Sul, 3: 161-180.

Durán Serrano, E. (2001): Hacia una legislación sobre la conservación del patrimonio cultural: normativas y conceptos. Revista de Arqueología Americana, 20: 131-142.

Dussel, E. (1983): Praxis latinoamericana y filosofía de la liberación. CLACSO, Buenos Aires

Dussel, E. (1994): 1492. El encubrimiento del Otro. Hacia el origen del "mito de la modernidad". UMSA, La Paz.

EcheverríA, J. (1998): Filosofia de la ciencia. Akal, Madrid.

Endere, M. L. (2001): Patrimonio arqueológico en Argentina. Panorama actual y perspectivas futuras. Revista de Arqueología Americana, 20: 143 - 158.

Endere, M.L. (2002): Arqueología, política y globalización ¿quién se ocupa del patrimonio arqueológico? Cuadernos (Facultad de Humanidades y Ciencias Sociales, San Salvador de Jujuy), 18: 77-89.

Endere, M.L. (2005): Talking about Others: Archaeologists, Indigenous Peoples and Heritage in Argentina. Public Archaeology, 4: 155 - 162 .

ENDERE, M. L.; RolANDI, D. (2007): Legislación y gestión del patrimonio arqueológico. Breve reseña de lo acontecido en los últimos 70 años. Relaciones. Número especial 70 años de la SAA, XXXII: 33 - 55.

Escobar, A. (1998): La invención del tercer mundo. Construcción y deconstrucción del desarrollo. Editorial Norma, Santa Fe de Bogotá.

FABIAn, J. (1983): Time and the others. How anthropology makes its object. Columbia University Press, Nueva York.

Fagan, B. (1972): In the beginning. An introduction to Archaeology. Brown and Co., Boston.

Fernández, A. C. (comp.) (2005): Foro con los pueblos originarios. Primer Congreso Latinoamericano de Antropología. Universidad Nacional de Rosario, Facultad de Humanidades y Artes, Santa Fe. 
Fernández Murillo, J. (2003): Comunidades locales y la enseñanza de la arqueología: una experiencia en Bolivia. Chungara, Revista de Antropología Chilena, 35(2): 355-359.

Funari, P. P. (1994): Rescuing Ordinary People's Culture: Museums, Material Culture and Education in Brazil. The presented Past. Heritage, Museum and Education (P. Stoney y B. Molyneaux, eds.). Routledge, Londres: 121-136.

FunAri, P.P. (2004): Arqueología Latinoamericana y su contexto histórico: La Arqueología Pública y las tareas del quehacer arqueológico. Hacia una arqueología de las arqueologías sudamericanas (A. Haber, ed.). Universidad de los Andes, Facultad de Ciencias Sociales, Centro de Estudios Socioculturales e internacionales, Uniandes, Bogotá: 83-90.

Funari, P.P.; Robrahn-GonzÁlez, E.M. (2006): Editorial. Arqueología Pública, 1:3.

Galison, P. (1991 [1999]). Trading zone. Coordinating action and belief. The Sciences Studies Reader (M. Biagioli ed.), Routledge, Nueva York: 137 - 160.

GÁndara VÁzquez, M. (1999): El estudio del pasado: explicación, interpretación y divulgación del patrimonio Cuadernos de Antropología Social, 5: 97 -124.

Gero, J. (2000): Why? and Whither? WAC. World Archaeological Congress Bulletin, 12: 55-63.

Gil García, F. (2005): Cuando vengan los turistas... ruinas arqueológicas, turismo y expectativas locales de futuro en Nor Lipez (Dpto. Potosí, Bolivia). Textos Antropológicos (Universidad Mayor de San Andrés, La Paz), 15(2): 197-228.

Gnecco, C. (2004): La indigenización de las arqueologías nacionalistas. Teoría Arqueológica en América del Sur (G. Politis y R. P. Peretti, eds.), Serie teórica 3, INCUAPA, Olavarría, Buenos. Aires: 119 - 128.

Godoy Gallardo, M. A.; Hernández Ojeda, J.A.; AdÁn Alfaro, L.I. (2003): Educación patrimonial desde el museo: iniciativas de promoción y puesta en valor del patrimonio cultural en la X Región. Revista Conserva, Centro Nacional de Conservación y Restauración de Chile, 7: 23-36.

Guraieb G.; Frère, M. (2008): Caminos y encrucijadas en la gestión del patrimonio arqueológico argentino. EUDEBA, Buenos Aires.

HABER, A.F. (1993): Hacia una comprensión de la construcción científica del pasado: ciencia y arqueología en el noroeste argentina. Alteridades, 3(6): 39-46.

HABER, A.F. (2004): Arqueología de la naturaleza, naturaleza de la arqueología. Hacia una arqueología de las arqueologías sudamericanas, (A.F. Haber, ed.), Universidad de los Andes, Facultad de Ciencias Sociales, Centro de Estudios Socioculturales e Internacionales, Bogotá: 15 -32.

Hall, M. (2004): Romancing the stones: archaeology in popular cinema. European Journal of Archaeology, $7: 159-176$.

Handler, R. (1996): Is identity a useful cross cultural concept? Commemorations. The politics of national identity (J. Gillis, ed.), Princeton University Press: 25-40.

Hernández Descalzo, P.J. (1997): Luces, cámara, jacción!: arqueología, toma 1. Complutum, 8: 311-334.

Hernando, A. (2006): Arqueología y globalización. Complutum, 17: 221-234.

HiRST, K. (1997): The roots of NAGPRA: An interview with Steve Russell. Disponible en: http://archaeology.about. com/cs/ethicsandlaw/a/russell.htm

Hobsbawn E.; Ranger, T. (1988 [2003]): The invention of tradition. Cambridge University Press, Cambridge.

Hochadel, O. (2009): Atapuerca - the Making of Magic Mountain. Popular Science books and human-origins research in Contemporary Spain. Comunicating science in 20th century Europe. A survey on Research and comparative perspectives (A. Schirrmacher, ed.), Max-Planck-Institute for the History of Science, Berlin: 149-163.

IAZzetA, O. (2008): Lo público, lo estatal y la democracia. Iconos. Revista de Ciencias Sociales (Facultad Latinoamericana de Ciencias Sociales-Sede Académica de Ecuador), 32:49-60.

Jofré, C. (comp.) (2010): El regreso de los muertos y las promesas del oro. Patrimonio arqueológico en conflicto. Encuentro Grupo Editor, Argentina.

Johnson, M. (2000): Teoría Arqueológica. Una introducción. Ariel, Barcelona.

Kojan, D.; Angelo, D. (2005): Dominant narratives, social violence and the practice of Bolivian archaeology. Journal of Social Archaeology, 5(3): 383-408.

Lahiri, N., Shepherd, N.; Watkins, J.; Zimmerman, L. (2007): Diálogos desde el sur/dialogos desde o sul. Foro Virtual: arqueología y descolonización. Arqueología Suramericana / Arqueologia Sul-americana, 3(1): 3-19. 
Lander, E. (2000): Diálogos a través del Atlántico Sur: saberes hegemónicos y saberes alternativos. Trabajo presentado en X Congreso Internacional de la Asociación Latinoamericana de Asia y África, Río de Janeiro, Brasil.

LANDER, E. (ed.) (2003): La colonialidad del saber: eurocentrismo y ciencias sociales. Perspectivas latinoamericana, CLACSO, Buenos Aires.

Lemaitre S.; Schall, C. (eds.) (2009): How do the media represent archaeology, what is at stake? Musées royaux d'Art et d'Histoire, Bruselas.

Lima TóRrez, M. Del P. (2003): Participación comunitaria, desarrollo sostenible y arqueología: el caso de Quila Quila (Chuquisaca, Bolivia). Chungara, Revista de Antropología Chilena, 35(2): 362-365.

LonDoÑo, W. (2003): Discurso jurídico versus discurso cultural: El conflicto social sobre los significados de la cultura material prehispánica. Boletín Museo del Oro, 51: 1-26.

LonDoÑo, W. (2007): Enunciados prescritos y no prescritos en arqueología: una evaluación. Boletín de Antropología de la Universidad de Antioquia, 21, N³8: 312-336.

Londoño, W.; Guatavita, E. (2003): El pasado en juego. Boletín Museo del Oro, 51. [URL: http://www.banrep. gov.co/museo/esp/boletin] Acceso: 15/03/2005.

López, C. E.; Reyes, M. (1994): The role of Archaeology in Marginalized Areas of Social Conflict: Research in the Middle Magdalena Region, Colombia. The presented past. Heritage, museus and education (P. Stone y B. Molyneaux, eds.). Routledge, Londres: 137- 147.

López MAzz, J. M. (2004): Arqueología e identidad uruguaya: el saber y el poder en las vanguardias intelectuales. Teoría arqueológica en América del Sur (G. Politis y R. Peretti, eds.), INCUAPA, Serie teórica No 3, Olavarría/ Buenos Aires: 197- 212.

Lowenthal, D. (1998): El pasado es un país extraño. Akal, Madrid.

Lumbreras, L. G. (1974 [1984]): La arqueología como ciencia social. Ediciones Peisa, Perú.

MacDonald, S.; Schaw, C. (2004): Uncovering ancient Egypt. The Petrie Museum and its public. En Merriman 2004: 110-131.

MANTEgaRi, C. (2002): Naturaleza y modernización en el siglo XIX: la expansión de la institucionalización científica. Saber y tiempo, 14: $11-31$.

MAPUndA, B.; LANE, P. (2004): Archaeology for Whose Interest - Archaeologists or the Locals? En Merriman 2004: 211-223.

Massa, L. (2008): Los niños de Llullaillaco y su exposición. Saqsaywaman (Revista del Instituto Nacional de Cultura, Cusco), 8:167-190.

Mazel A.; Ritchie, G. (1994): Museums and their messages: the display of the pre and early colonial past in the museums of South Africa, Botswana and Zimbabwe. The Presented Past: Heritage, museums and education (P. G. Stone y B. L. Molyneaux, eds.), One World Archaeology. Routledge. Londres: 225-235.

McDAVID, C. (2004): Towards a more democratic archaeology? The Internet and public archaeological practice. En Merriman 2004: 159-187.

McGimsey, Ch. (1972): Public archaeology. Seminar Press, Nueva York.

McGuire, R. H.; NAVARRETe, R. (1999): Entre motocicletas y fusiles: las arqueologías radicales anglosajonas e hispanas. Anais da I Reuniao Internacional de Teoria Arqueológica na América do Sul. Revista do Museu de Arqueologia e Etnologia, (Universidade de Sao Paulo), Suplemento 3:181-199.

McNiven, I. J. C.; Russell, L. (2005): Appropriated past. Indigenous peoples and the colonial culture of Archaeology. Altamira Press, Walnut Creek.

Meneses Pacheco, L. (2010): La arqueología venezolana de fines del siglo XIX y comienzos del siglo XX. En Nastri y Ferreira 2010: 21-54.

Merriman N. (2002): Archaeology, heritage and interpretation. Archaeology: the Widening Debate (B. Cunliffe, W. Davies y C. Renfrew, eds.), Oxford University Press/British Academy, Oxford: 541-566.

Merriman, N. (ed.) (2004): Public Archaeology. Routledge, Londres.

Mignolo, W. (1995). The darker side of the Renaissance: Literacy, Territoriality, and Colonization. University of Michigan Press.

Mignolo, W. (2003): La colonialidad a lo largo y a lo ancho: el hemisferio occidental en el horizonte colonial de la modernidad. En Lander 2003: 55-86. 
Minteguiaga, A. (2008): Lo público: Estado y sociedad civil en América Latina. Iconos. Revista de Ciencias Sociales (Facultad Latinoamericana de Ciencias Sociales-Sede Académica de Ecuador), 32: 33-36.

Molinari, R. (2000): ¿Posesión o participación? El caso del Rewe de la comunidad mapuche Ñorquinco. II Congreso Virtual de Antropología y Arqueología, www.naya.org.ar.

Moreno de Giraldo, E. (1994): The Colegio Nueva Granada Arqueological Museum, Colombia: a proposal for the Development of Educational Museums in Schools. The presented past. Heritage, Museus and Education, (P. Stone y B. Molyneaux, eds.) Routledge, Londres: 159-171.

NASTRI, J. (2005): La construcción arqueológica del pasado. Los primeros americanistas (1876-1926) y la recuperación de las culturas indígenas de los valles calchaquíes. Tesis de Maestría en Sociología de la Cultura y Análisis Cultural, Universidad Nacional de General San Martín Instituto de Altos Estudios Buenos Aires.

NAStri, J.; Ferreira, M. (eds.) (2010): Historias de la Arqueología Sudamericana. Fundación de Historia Natural Félix da Azara, Buenos Aires.

Nielsen, A., Calcina, J.; Quispe, B. (2003): Arqueología, turismo y comunidades originarias: una experiencia en Nor Lípez (Potosí, Bolivia). Chungara, 35(2): 369-377.

NuÑEz, L. (2006): Para conservar el alma patrimonial. VIII Seminario sobre Patrimonio Cultural. Rescate, invención y comunidad. Dibam, Consejo Nacional de la Cultura y las Artes, Consejo de Monumentos y Universidad Arcis, Santiago de Chile: $62-75$.

OdAK, O. (1985): Las raíces del futuro. Correo de la UNESCO, Julio: 9-11.

Onetto M., Cassiodoro, G.; Colombo, M.; Salerno, V.; Elías; A.; Castro, A. (2008): Imagínatelo en vivo: patrimonio en acción y arqueología todo terreno en Cueva de las Manos, Santa Cruz. Cuadernos del Instituto Nacional de Antropología y Pensamiento Latinoamericano, 22: 145-154

Parker Pearson, M.; Ramilisonina (2004): Public archaeology and indigenous communities. En Merriman 2004: $224-239$.

Pegoraro, A. (2009): Las colecciones del museo etnográfico de la Universidad de Buenos Aires: un episodio de la historia del americanismo en la Argentina. Tesis de doctorado de la Facultad de Filosofía y Letras, Universidad de Buenos Aires.

PiAzZinI, C. E. (2001): Arqueología, legislación y desarrollo: Balance de los noventa y perspectivas desde la coyuntura actual. Arqueología, patrimonio y sociedad (D. Patiño C., ed.), Universidad del Cauca, Colombia: 23-40.

PIÑon, A. (2008): Brasil. Arqueología, identidad y origen. Ediciones Suárez, Argentina.

Podgorny, I. (1999): Arqueología de la Educación. Textos, indicios, monumentos. La imagen de los indios en el mundo escolar. Sociedad Argentina de Antropología, Buenos Aires.

Podgorny, I. (2004): Tocar para creer. La arqueología en la argentina, 1910 - 1940. Anales del Museo de América, 12: $147-182$.

Podgorny, I. (2009): El sendero del tiempo y de las causas accidentales. Los espacios de la prehistoria en la Argentina, 1850-1910. Protohistoria, Rosario, Argentina.

Politis, G. (1995): The Socio-politics of the Development of Archaeology in Hispanic South America. Theory in archaelogy. A world perspective, (P. Ucko, ed.). Routledge, Londres: 197-235.

Pupio, M. A. (2005): Coleccionistas de objetos históricos, arqueológicos y de ciencias naturales en museos municipales de la provincia de Buenos Aires en la década de 1950. História, Ciências, Saúde-Manghinos. Dosier Museos y Ciencias, 12: 205-229.

Pupio, M. A.; Visotsky, J. (2001): Museo y escuela: algunas experiencias para redefinir los contenidos de esta relación. Actas del I Encuentro Iberoamericano Museos y Centros Históricos, Comunidad y Educación, La Habana: 28 - 30.

Querol, M. A. (2001): De maravillosos hombres y pobres monos. Análisis del fenómeno antropocentrista en la bibliografía española sobre los orígenes humanos. Complutum, 12: 237-248.

Quintana, C. (comp.) (1999): Escuela y sociedades indigenas. Análisis de experiencias de extensión universitaria sobre la arqueología regional. Universidad Nacional de Mar del Plata y Municipalidad de General Pueyrredón, La Plata.

Ramos, M. (2009): Etnocidio y genocidio. Nosotros" y los "otros". Ciencias Sociales. Líneas de acción didáctica y perspectivas epistemológicas (M. Insaurralde, coord.), Editorial Novedades Educativas, Buenos Aires, México: 49-192. 
Rance, S.; Salinas Mulder, S. (2001): Investigando con ética: aportes para la reflexión-acción. CIEPP y Population Council, La Paz.

Ratto, N. (2002): Patrimonio arqueológico y megaproyectos mineros: el impacto arqueológico en detrimento de su potencial para el desarrollo sostenido regional en la provincia de Catamarca (Argentina). Tesis Master en Estudios Ambientales (UCES).

Richardson III, J. B. (2001): Recuperando el Perú precolombino: investigación arqueológica versus tesoro, saqueo y botín. Revista de Arqueología Americana, 20: 31-50.

RocA, A. (2008): Objetos alheios, histórias compartilhadas: os usos do tempo em um museu etnográfico. Minc. IPHAM, DEMU, Río de Janeiro.

Romanos De TiRATEL, S. (2008): Revistas argentinas de humanidades y ciencias sociales. Visibilidad en bases de datos internacionales. Instituto de Investigaciones bibliotecológicas, Facultad de Filosofía y Letras, Universidad de Buenos Aires, Buenos Aires.

Ruiz ZAPATERo G. (2002): Arqueología e identidad: la construcción de referentes de prestigio en la sociedad contemporánea. Arqueoweb, 4(1). [URL: http://www.ucm.es/info/arqueoweb/pdf/4-1/ruizzapatero.pdf] Acceso el 4/06/2006

SaldañA, J. J. (1993): Nuevas tendencias en la historia latinoamericana de la ciencia. Cuadernos Americanos (UNAM, Centro de Investigaciones sobre América Latina y el Caribe), 2(38): 69 - 91.

SAlerno, V. M. (2011-2012): Trabajo arqueológico y representaciones del pasado prehispánico en Chascomús. Tesis Doctoral, Facultad de Filosofía y Letras, Universidad de Buenos Aires (inédita).

SAlerno, V.; Pupio, A. (2009): Más allá de lo que se escribe: la arqueología como metáfora en los medios gráficos. Arqueología Suramericana - Arqueología sul-americana, 2(2): 153-177.

SAlomón, J.J. (2008): Los cientificos. Entre poder y saber. Editorial de la Universidad Nacional De Quilmes, Buenos Aires.

Saucedo-Segami, D. (2006): Arqueología pública y sus posibilidades en el Perú. Arkeos, Revista Electrónica de Arqueología (Pontificia Universidad Católica del Perú), 1(1).

Schadla-Hall, T. (1999): Editorial: Public archaeology. European Journal of Archaeology, 2(2): 147-158.

SEgobye, A. (2006): Historias Estratificadas en el desarrollo de la Arqueología Pública en el Sur de África. Arqueología Suramericana - Arqueología sul-americana, 2(1): 93-118.

TANTAlé́n, H. (2010): El pasado tras del espejo: arqueología y nacionalismo en el Perú. En Nastri y Ferreira 2010: 137-166.

TÉllez, F. (2002): Museología y patrimonio, una propuesta de educación interactiva tangible. Cuadernos (Revista de la Facultad de Humanidades y Ciencias Sociales, San Salvador de Jujuy), 18: 91-109.

Trigger, B. (1992): Historia del pensamiento arqueológico. Crítica, Barcelona.

UCKO, P. (1994): Museums and sites: cultures of the past within education - Zimbabwe some ten years on. The Presented Past: Heritage, museums and education (P. G. Stone y B. L. Molyneaux, eds), One World Archaeology, Routledge, Londres: 237-278.

Vara A. M.; Hurtado de Mendoza, D. (2004): Comunicación pública, historia de la ciencia y 'periferia'. Certezas y controversias. Apuntes sobre la divulgación científica, Libros del Rojas, Universidad de Buenos Aires, Buenos Aires: 71-103.

Vargas Arenas, I. (1990): Arqueología, ciencia y sociedad. Editorial Abre Brecha, Caracas.

Verdesio, G. (2004): La mudable suerte del amerindio en el imaginario uruguayo: su lugar en las narrativas de la nación de los siglos XIX y XX y su relación con los saberes expertos. Hacia una arqueología de las arqueologías sudamericanas, (A.F. Haber, ed.) Universidad de los Andes, Facultad de Ciencias Sociales, Centro de Estudios Socioculturales e internacionales, Ediciones Uniandes, Bogotá: 115-150.

Wolf, E. (1987 [2000]). Europa y la gente sin historia. Fondo de Cultura Económica, Buenos Aires. 